\title{
LAURE LASSAGNE, Ce que se parler veut dire. La représentation du monologue dans les romans de Stendhal
}

\section{Michel Arrous}

\section{(2) OpenEdition}

Journals

\section{Édition électronique}

URL : https://journals.openedition.org/studifrancesi/31858

DOI : 10.4000/studifrancesi.31858

ISSN : 2421-5856

Éditeur

Rosenberg \& Sellier

\section{Édition imprimée}

Date de publication : 1 août 2020

Pagination : 413-414

ISSN : 0039-2944

\section{Référence électronique}

Michel Arrous, "LAURE LASSAGNE, Ce que se parler veut dire. La représentation du monologue dans les

romans de Stendhal », Studi Francesi [En ligne], 191 (LXIV | II) | 2020, mis en ligne le 01 septembre 2020, consulté le 18 septembre 2021. URL : http://journals.openedition.org/studifrancesi/31858; DOI : https://doi.org/10.4000/studifrancesi.31858

Ce document a été généré automatiquement le 18 septembre 2021.

\section{(c) $(1) \odot$}

Studi Francesi è distribuita con Licenza Creative Commons Attribuzione - Non commerciale - Non opere derivate 4.0 Internazionale. 


\title{
LAURE LASSAGNE, Ce que se parler veut dire. La représentation du monologue dans les romans de Stendhal
}

\author{
Michel Arrous
}

\section{RÉFÉRENCE}

LAURE LASSAGNE, Ce que se parler veut dire. La représentation du monologue dans les romans de Stendhal, Paris, Honoré Champion, 2016, 483 pp.

1 «Qu'est-ce donc que se parler veut dire?». Poser cette question sur le dédoublement réflexif autonome (sans rapport avec le modèle de la confession), c'est chercher à comprendre le choix que fait Stendhal romancier dans les modalités d'appréhension et de représentation du fait subjectif. Bien qu'il ne soit pas le seul mode narratif utilisé par Stendhal, le discours intérieur est un tropisme stendhalien évident (on pense aussitôt au monologue de Julien sous le grand tilleul de Vergy, ou à son long monologue en prison) et fréquent (voir le relevé aux pp.443-450), mais aussi paradoxal puisque le personnage «se dit» ce qu'il connaît déjà, sous la forme d'un dialogue fictif par lequel s'élabore la pensée dans une activité réfléchie.

2 Dans l'introduction de son ouvrage passionnant issu d'une thèse de doctorat (Paris IV, 2007), Laure Lassagne rappelle que Paul Bourget fut le premier, en 1882, soit peu de temps avant que ne s'installe le mythe de Stendhal intimiste, à identifier le monologue comme une technique de narration propre à Stendhal. Si le discours intérieur ou plutôt la «parole intérieure» a fait l'objet de nombreuses études de la part des psychologues, linguistes et philosophes, force est de constater que dans le cas de Stendhal c'est l'écriture de soi et le récit à la première personne qui ont monopolisé l'attention, au détriment de l'étude des modes de narration de la vie intérieure dans les romans; néanmoins Prévost avait dit à propos du Rouge que «presque toujours nous sommes dans l'âme du héros». D'où l'intérêt de la présente étude systématique, d'autant plus 
qu'on ne disposait jusqu'à sa parution que d'un article de Dominique Trouiller (Le monologue intérieur dans "Le Rouge et le Noir", "Stendhal Club", 1969) et de la thèse d'Annie Sbarge (1996) sur le monologue intérieur dans la Chartreuse dont quelques pages figurent dans "L'Année stendhalienne" de 1997. L. Lassagne aurait pu aussi mentionner l'étude que J.T. Booker a donnée du style direct libre chez Stendhal en 1985. À vrai dire, c'est bien avant Bourget que cette technique du monologue a été remarquée puisque les premiers lecteurs du Rouge ont parfois reproché à Stendhal une volonté perverse de manier la loupe et le scalpel, mais l'on ne parlait pas encore de «monologue intérieur». Ajoutons que l'expression figure dans Charles Demailly des Goncourt, en 1859, avant que Bourget ne l'emploie dans Cosmopolis. Le procédé connut son heure de gloire dans les années 1920-1930, quand Édouard Dujardin s'en attribua la paternité, en prétendant, contre toute évidence, que Stendhal ne l'avait pas utilisé. Pour analyser la spécificité stendhalienne de ce mode d'écriture, L. Lassagne, à la différence de Dujardin, a dissocié le niveau de la réalité psychique (le surgissement de la pensée) du niveau de la transcription ou de la représentation selon les conventions littéraires du XIX ${ }^{e}$ siècle. On sait que Stendhal n'a cessé de s'adonner à la «connaissance du cœur de l'homme» et qu'il a explicitement déclaré à Balzac: «Je veux parler de ce qui se passe au fond de l'âme de Mosca, de la duchesse, de Clélia».

3 Dans la première partie, de rigoureuses analyses stylistiques - voir, par exemple, le cas des verbes introduisant le discours intérieur-expliquent les règles du dispositif énonciatif et le fonctionnement du discours intérieur. Chez Stendhal, dont est opportunément rappelée la réticence, finalement surmontée, au recours à la première personne, le monologue, au lieu d'être construit sur le modèle du monologue dramatique (lequel fait chez lui office de repoussoir), est une situation énonciative originale dans laquelle le narrateur intervient, parfois de manière indiscrète, en faveur du lecteur, ne serait-ce que pour dénoncer la mauvaise foi du personnage. Dans le chapitre 2 consacré à la configuration des monologues romanesques où le narrateur pratique l'oscillation entre le je et le $i$, on suit l'élaboration du dispositif, dans Armance («la formulation d'un secret dans une conscience»), puis sa mise en scène dans le Rouge et Lucien Leuwen.

La deuxième partie traite de l'imagination linguistique de Stendhal. L. Lassagne effectue alors un nécessaire détour par l'Idéologie, non sans noter les infléchissements que Stendhal fait subir à la pensée de Tracy (à propos de ce dévoiement, la première partie de Raison et déraison chez Stendhal de Michel Crouzet aurait dû être mentionnée), pensée librement interprétée, notamment la réappropriation du langage, l'acquisition d'une parole apte à affirmer l'originalité du moi, tout en refusant la stylisation romantique ou le lyrisme façon Chateaubriand. Cette théorie du langage, Sten-dhal la met en œuvre dans l'énoncé des monologues romanesques (chap. 2), mais la rigueur recherchée ne va pas sans quelques flottements ou approximations: il n'est pas rare d'y trouver des tours communs, des formules lexicalisées, des citations, des métaphores faciles, des clichés (Mathilde est une championne du cliché et Julien de la grandiloquence littéraire), et même des stéréotypes dignes des «romans pour femmes de chambre». D'où les sarcasmes du narrateur à l'égard des héros. L. Lassagne conclut en notant que chez Stendhal «le personnage ordonne ses expériences, donne forme et vigueur à ses sentiments en recourant en priorité à des paradigmes littéraires». Cette dramatisation énonciative est étudiée dans le détail syntaxique et la tonalité de la voix intérieure. L'analyse des marqueurs du «style intérieur» confirme la «tendance ratiocinante» des personnages - on pensera à l'asthénie d'Octave ou à la paralysie de 
Lucien qui ne sait pas ce qu'il veut - qui pratiquent tous et toujours un véritable art du récit dans la narration de soi.

Intitulée «Enjeux du monologue stendhalien», la troisième partie abandonne l'approche psycho-linguistique au profit de l'analyse stylistique et littéraire du discours intérieur, plus précisément l'analyse des effets sur le lectorat. Dans la narration des états de l'intimité subjective, Stendhal a mis en place un ensemble de stratégies dans lesquelles il s'oppose aux modes littéraires contemporaines. Des exemples plus nombreux auraient sans aucun doute permis de mieux évaluer ce déni de communication chez celui qui a pris le parti de ne pas tout dire. Les monologues offrent du sujet une représentation réflexive qui permet au lecteur d'appréhender son activité intérieure dans sa totalité psychique et politique, dans ses rapports avec lui-même et avec la société. Dans les procédés utilisés pour rendre compte de la vie intérieure, il est parfois difficile de distinguer la pensée du personnage de celle de l'auteur - Leo Spitzer en a donné un bel exemple en 1959 dans son étude sur l'intensif «si»-car le roman stendhalien brouille les frontières, comme le prouvent ces cas fréquents où l'on passe subrepticement, par un effet de fondu, du récit au monologue rapporté au style direct libre, du psycho-récit au monologue narrativisé, à tel point que le lecteur peut se demander qui parle. Enfin, et cet aspect est souligné à juste titre, dans le monologue tel que le pratique Stendhal, le sujet s'affirme dans une vision orientée par l'esprit d'examen et l'insurrection contre l'autorité parentale, sociale, cléricale. C'est bien sûr le cas de Julien et de Lamiel. L. Lassagne a raison d'attirer l'attention sur la valeur polémique et politique des monologues qui participent ou contribuent à la révolution libérale.

6 Mise en récit de l'existence, le monologue stendhalien dont la complexité est méthodiquement et clairement exposée dans tous ses infléchissements, et sans les excès de la narratologie, joue donc un rôle essentiel, non seulement dans la représentation de la vie intérieure des personnages que découvre le lecteur, mais aussi parce qu'il permet d'accéder à l'intériorité de l'individu aux prises avec la réalité de la vie sociale. 